\title{
Efficient Approach for Rapid Sample Protocol in Wireless Scenario
}

\author{
MadhulikaMalviya \\ LNCT, Indore
}

\author{
Mr. JitendraDangra \\ LNCT,Indore
}

\author{
M. K Rawat, PhD. \\ LNCT, Indore
}

\begin{abstract}
This paper presents an Optimized Rapid Sample Protocol based on Clustering Approach along with comparative results between Rapid Sample and its optimized version . In the past, under various protocol and rate adaption technique intense research has been done to adapt best bit rate for improving performance of wireless network through analysis of channel behavior. In the journey of development for achieving improved performance of wireless network, rapid sample protocol has been designed to elevate network performance over frequent channel variation scenario. Implementation of this work is segregated in three parts, At initial level ,existing Rapid Sample Protocol is implemented and values of the considered parameters are recorded, in next level using clustering concept optimization of Rapid Sample Protocol has been done and in final stage comparative results between initial two levelis provided by plotting various graphs for individual parameters like Packet loss, Throughput and Packet Delivery Ratio.
\end{abstract}

\section{General Terms}

Wireless Network, Rate Adaptation

\section{Keywords}

Optimization, Performance, Rapid Sample Protocol.

\section{INTRODUCTION}

\subsection{Overview}

It can be projected that progressively more 802.11 devices are used by mobile consumers, reason being fast and hectic lifestyle of this era. According to a research report of CISCO , monthly global mobile data traffic will surpass 10 Exabyte in year 2017 as compared to 885 Petabytes per month in 2012 [8] .So it can be easily analyzed that usage of such devices will increase tremendously in upcoming years. Main focus is required at the shortcomings of wireless scenario, so that communication becomes more prominent in mobility mode also. As mobility factor is introduced into the picture, problem of losses and unstable channel condition like fading due to distance, interference etc arises.

To resolve this issue and upgrade the performance of wireless network, bit rate adaptation [5 ] technique was incorporated which measures or analyze channel behavior and provide best bit rate for transmission accordingly, which result in improved throughput and minimized packet loss. Rate adaptation is based on three types of schemes, i.e. SNR based, Frame based and Hybrid .In the next section of background, detailed view of rate adaptation schemes will be provided.

Similarly, variable data rate or rate adaptation technique is also integrated in wireless sensor network as major issue with this kind of network is related with power consumption and latency. It has been observed that variable rate for communication in wireless sensor network, provides reduced energy consumption, which in turn improve efficiency [3] . These are used in wireless sensor network for build sensor system that adapt to network and environment dynamics and to get better performance as compared with single data rate communication, which is used in most of the wireless senor network protocols.

\subsection{Report Organization:}

The flow of the research paper is as follows. Section 1 is titled as Introduction which describe objective and the motivation behind this work. Background work is section 2 which is domain on the basis of which this particular research work arises. Next part describes the basic theory of existing approach for Rapid Sample Protocol and Proposed approach for the same. Section 4 is of implementation work that includes operating environment, simulation tool overview and snapshots of execution process. Section 5 gives comparative values between two protocol's on the basis of quality of service parameters. Section 6 draws conclusion from the work described in the previous sections. Future section discusses possibilities for further development and application area

\section{BACKGROUND WORK:}

As concluded in the last section, rate adaptation techniques are desirable for wireless sensor networks, to get best result, so here discussion will be continued on various scheme under rate adaptation [5 ]. In the end a brief overview of Hint Aware Bit Rate Adaptation is also provided.

\subsection{Classification Of Rate Adaptation Scheme:}

Rate adaptation is actually determination of best data transmission rate favorable for existing channel condition. Data rate is decided according to current scenario so classification is equally important, which can be done either on the basis on technique applied or on the basis of loss differentiation.

\subsubsection{Classification based on technique [7 ]:}

First category is SNR based rate adaptation [7 ], in which sender of packet uses feedback from receiver about Signal to Noise ratio . One odd point is this scheme requires expensive and constant feedback from receiver and it is inaccurate because channel condition may change since feedback was received. Second one is Frame based rate adaptation [7 ] in which channel strength is analyzed by past pattern of packet delivery success. Here probe packets are sent before actual transmission which is very expensive approach. Last is hybrid rate adaptation[2 ] which is actually combination of above discussed two schemes. This approach is useful for real time streaming applications. 


\subsubsection{Classification based on loss differentiation}

\section{[7]:}

Loss differentiation is a basic term which reflects the cause behind the losses occurred during transmission of data [14]. Losses canbe due to channel degradation or collision, and if reason behind this is not cultivated, rate adaptation scheme will be highly inefficient. Most common Protocol that make a distinction of the cause is CARA i.e. Collision Aware Rate Adaptation which sends RTS/ CTS frames before retransmission of loss frame and if RTS frame is also lost it will consider collision as main cause behind this.

\subsection{Hint Aware Bit Rate Adaptation [1]}

Traditionally all the wireless protocol uses information such as bit error, SNR to adapt their behavior, later on Hint Aware Protocol Architecture [1 ] was designed that augments these information with the hints from external sensor of mobile phones, tablets etc which gives improved performance of the system. After that Hint Aware Bit rate adaptation scheme [1 ] was launched by group of researchers at MITs, in which different approach is opted depending upon whether nodes moving or static. This concept was introduced after a couple of experiment done in static and mobile cases, which concludes that in mobile settings fate of upcoming packets depends highly on previous packet which is opposite in case of static. In mobile scenario channel conditions are volatile so it no logic to maintain long record of performance at different data rate whereas in static case it does maintain long history. With these points in mind Hint Aware Bit Rate Adaptation Protocol was implemented that uses two frame based rate adaptation protocols, which are switched on the basis of movement hints from receiver. At the Sender Rapid Sample Protocol [1] is used when nodes are moving and Sample Rate [ 4] is opted when nodes are static. This information about current state of the device is given by receiver using external hints.

\section{RAPID SAMPLE PROTOCOL :}

As this work rely on performance enhancement of Rapid Sample Protocol for bit rate adaptation, so here need arises for basic discussion on same. In the end brief overview is also given for optimized approach opted.

\subsection{Rapid Sample Protocol based on rate adaptation technique [1]:}

This is a frame based rate adaptation protocol, which starts transmission with fastest bit rate and if acknowledgment is not received it will switch to lowest rate. Rapid Sample Protocol is particularly designed for channels with frequent deviations. Every point when packet is sent its time is recorded either as success time or failure time. Two basic parameters are considered over here $\delta$ success and $\delta$ failure [1].

If at particular bit rate successful acknowledgment is received, it will sample at this rate for $5 \mathrm{~ms}$ which is success value. Similarly this protocol has defined the value of failure as $10 \mathrm{~ms}$, which means if sampling is to be done on any previously failed rate it needs to wait for $10 \mathrm{~ms}$.

\subsection{Optimized Approach for Rapid Sample Protocol:}

Rapid Sample Protocol is meant of rapidly changing environment as channel varies quickly due to which frequent sampling is required, so it must gives good performance when nodes moves. Rate adaptation scheme desires to achieve higher bit rate for transmission so that communication take place quickly. And higher bit rate is only achieved when packet get delivered successful because when drop occurs bit rate is lowered. So, to improve the performance of Rapid Sample Protocol our main aim is to reduce Packet drop. Main factor behind these drops or losses is rapidly changing position of nodes. To sort out this issue and elevate the overall performance concept of clustering [6]is applied into current scenario, whole network is conceptually divided into clusters of static, mobile nodes. If one mobile node wants to send data to another mobile node, then chances of packet loss is high, but if a static node is used to transfer this packet to second mobile node then packet loss can be eliminated. Similar concept is used in VANET i.e. vehicular Adhoc Network [13] in which Road Side Units (RSU) are used to transfer signaling to vehicle in the network. [9 ]. In this way this approach can result is decreased packet loss, increased packet delivery ratio and hence overall throughput of the system. . A detailed Architecture for optimized Rapid Sample Protocol is depicted in [12] .

\section{IMPLEMENTATION}

This section presents detailed and representative performance evaluation of Rapid Sample Protocol and Optimized Rapid Sample protocol using ns-3 network simulator [11]. Additionally a comparative result is provided between both the version of this protocol, by plotting comparative graphs using Gnuplot

\subsection{Simulation Configuration:}

To prepare simulation for desired network utility we provide the above given simulation setup. Table 1 shows the exact simulation scenario by considering factors i.e. no of nodes, simulation time etc

Table 1.Showing Simulation setup for implementation of Proposed work.

\begin{tabular}{|l|l|l|}
\hline $\begin{array}{l}\text { Simulation } \\
\text { properties }\end{array}$ & Values & Description \\
\hline Channel Type & $\begin{array}{l}\text { Wireless } \\
\text { Channel }\end{array}$ & $\begin{array}{l}\text { This list of } \\
\text { WLAN channels } \\
\text { is the set of } \\
\text { legally allowed } \\
\text { Wireless LAN } \\
\text { channels using } \\
\text { IEEE 802.11. }\end{array}$ \\
\hline $\begin{array}{l}\text { No of Mobile } \\
\text { Nodes }\end{array}$ & 10 to 100 & $\begin{array}{l}\text { Nodes in the } \\
\text { simulation } \\
\text { presents the } \\
\text { devices that } \\
\text { behaves like } \\
\text { actual mobile } \\
\text { devices and base } \\
\text { stations }\end{array}$ \\
\hline
\end{tabular}




\begin{tabular}{|l|l|l|}
\hline Simulation Time & 50 & $\begin{array}{l}\text { The total time } \\
\text { when the } \\
\text { simulation is } \\
\text { visible and } \\
\text { locating its } \\
\text { positions. }\end{array}$ \\
\hline $\begin{array}{l}\text { Movement of } \\
\text { nodes }\end{array}$ & $\begin{array}{l}\text { Random } \\
\text { movement }\end{array}$ & $\begin{array}{l}\text { There is no } \\
\text { more specific } \\
\text { direction for } \\
\text { movement of } \\
\text { nodes. }\end{array}$ \\
\hline
\end{tabular}

\subsection{Simulation Tools [11]:}

To reflect the real world scenario network simulators are used , here to implement Rapid Sample protocol ns-3[11 ] is used along with other utilities. Ns-3 is discrete event simulator and its main target is networking research. Ns-3 provides substantial support for simulation of TCP, routing, and multicast protocols over wired and wireless networks. A simulator model of a real-world system is necessarily a simplification of the real-world system itself. The general procedure of creating a replication can be divided into several steps.

4.2.1 Topology definition: To ease the creation of basic facilities and define their interrelationships, ns-3 has a system of containers and helpers that facilitates this process.

4.2.2. Model usage: Models are added to simulation (for example, UDP, IPv4, point-to-point devices and links, applications); most of the time this is done using helpers.

4.2.3 Node and link configuration: Models set their default values (for example, the size of packets sent by an

pplication or MTU of a point-to-point link); most of the time this is done using the attribute system.

4.2.4 Execution: Simulation facilities generate events, data requested by the user is logged.

4.2.5 Performance Analysis: After the simulation is finished and data is available as a time-stamped event trace. This data can then be statistically analyzed with tools like R to draw conclusions.

4.2.6 Graphical Visualization: Raw or processed data collected in a simulation can be graphed using tools like Gnu plot.

\subsection{Experimental Scenario:}

In this experiment, implementation of Rapid Sample Protocol is done on ns-3 and visual effect can be seen on Net Anim animator [11]. Figure 1 shows screenshot of experimental setup of Rapid Sample Protocol.

After complete analysis of considered parameters like throughput, PDR, packet drop and end to end delay, next step is to implement Optimized Rapid Sample Protocol on the basis of clustering approach in ns-3 and visual scenario is prepared on Net Anim. Figure2 depicts experimental setup of optimized Rapid Sample Protocol. This new approach will result in improved performance of protocol

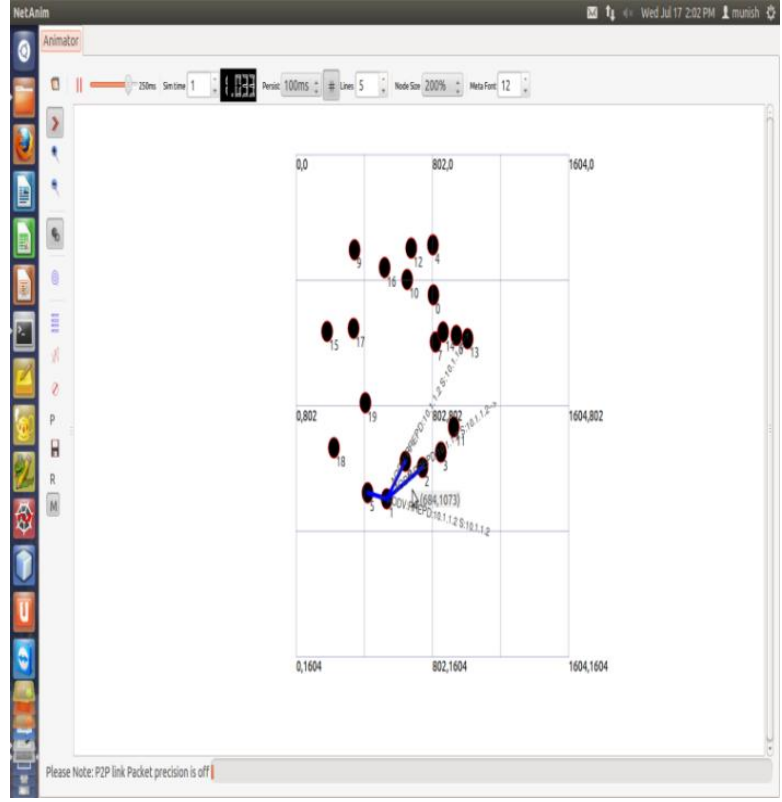

Figure 1: Snapshot showing setup of Rapid Sample Protocol in Wireless Scenario.

Figure 1 shows mobility of nodes and communication in reference of Rapid Sample Protocol. Whereas figure2 depicts scenario for optimized approach of Rapid Sample Protocol.

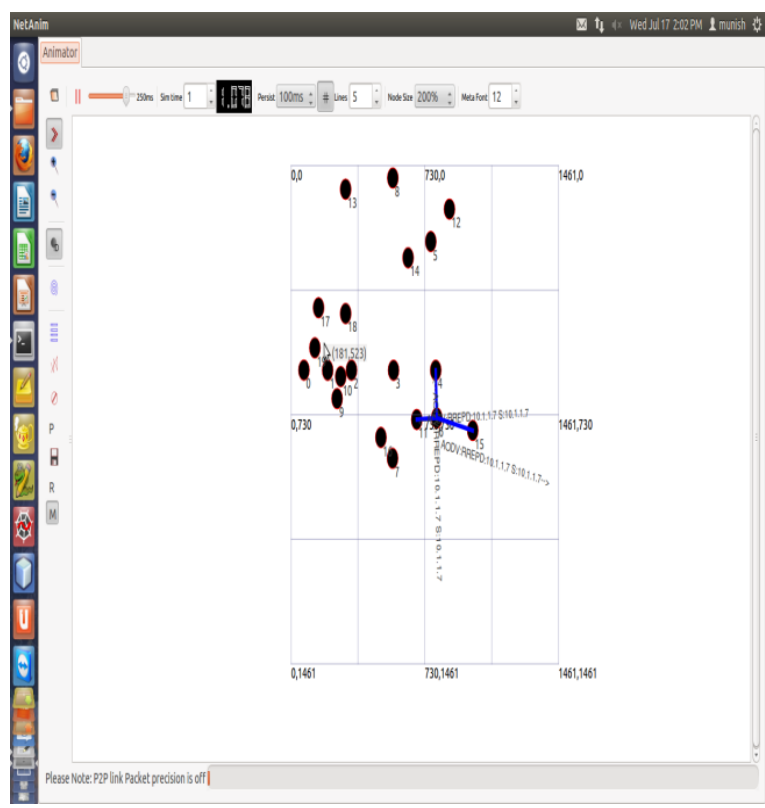

Figure 1: Snapshot showing setup of Optimized Rapid Sample Protocol in Wireless Scenario

\section{EXPERIMENTAL RESULT}

Based on the detailed analysis done in the last section i.e. on Rapid Sample Protocol and Optimized version of it, here a brief summary is given in tabular format (Table2) that includes the values of various measured parameters under diverse network formations. Table 2 depicts result of both the protocols. Here three quality of service parameters are considered for evaluation. As performance of any protocol depends on Throughput and Packet Delivery ratio, so these are opted here for analysis. 
To add visibility to the scenario, here comparative graphs are plotted in Gnuplot [10] for individual parameters showing the overall elevated performance. Figure 3 shows comparative graph for dropped packets, which is actually no of packet lossed during that particular instance of time. Whereas Figure 4 shows comparative values for Throughput and figure 5 shows comparative values of PDR i.e. Packet Delivery Ratio which is no of successful packets per hundred.

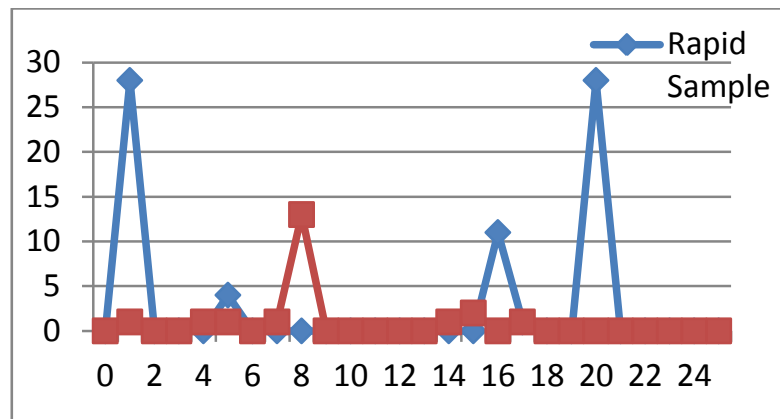

Figure3: Graph showing comparative values for Dropped Packets

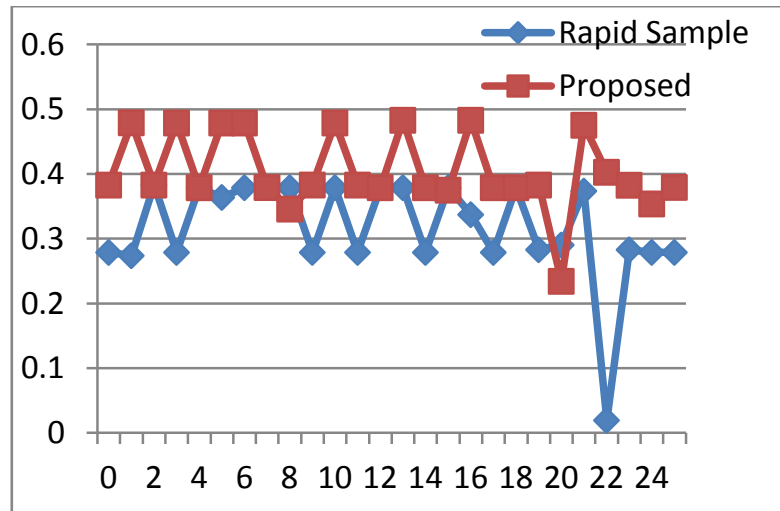

Figure4 : Comparative graph showing Throughput values obtained for both approaches.

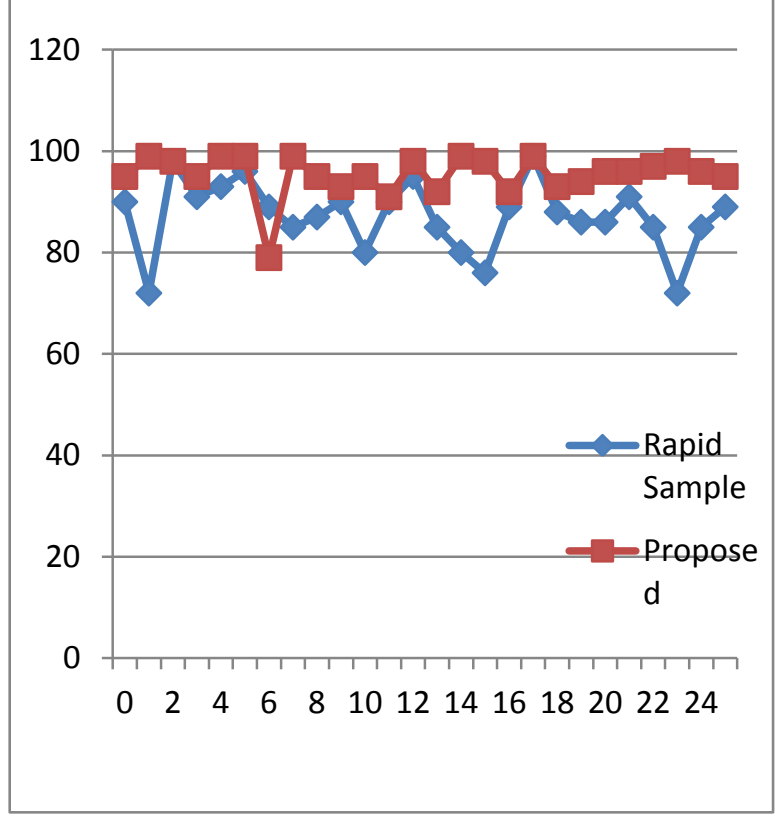

Figure 5: Showing Comparative graphs for Packet Delivery Ratio.

\section{CONCLUSION}

Through this paper we have analyzed performance of existing rapid sample protocol which is already employed under Hint Aware Bit Rate Adaptation Protocol. With the use of clustering concept we have proposed an optimized rapid sample protocol. This approach uses static nodes for data transfer between two mobile nodes so as to limit packet loss, which is turn result in increased packet delivery ratio. And hence overall performance will be increased.

By study of various comparative graphs and associated results we can conclude that by proposed approach in existing Rapid sample protocol performance and overall efficiency of the network.

Table 2:Comparative Result Summary of Rapid Sample Protocol and Optimized version

\begin{tabular}{|l|l|l|l|l|l|l|l|l|}
\hline No. of Nodes & Sink Node & $\begin{array}{l}\text { Source } \\
\text { Node }\end{array}$ & & \multicolumn{3}{|l|}{ Rapid Sample Protocol } & \multicolumn{3}{l|}{ Optimized Rapid Sample Protocol } \\
\cline { 5 - 10 } & & & $\begin{array}{l}\text { Th. Put } \\
\text { (Kbps) }\end{array}$ & PDR & $\begin{array}{l}\text { Av. } \\
\text { P.drop }\end{array}$ & $\begin{array}{l}\text { Th. Put } \\
\text { (Kbps) }\end{array}$ & PDR & P.drop \\
\hline 20 & 3 & 5 & 6 & 94 & 17 & 10 & 99 & 4 \\
\hline 40 & 6 & 4 & 4 & 90 & 5 & 14 & 96 & 5 \\
\hline 35 & 4 & 1 & 2 & 97 & 19 & 18 & 99 & 1 \\
\hline 50 & 9 & 12 & 2 & 96 & 2 & 7 & 97 & 1 \\
\hline
\end{tabular}




\section{ACKNOWLEDGMENTS}

I Madhulika Malviya, would like to acknowledge and extent my gratitude to my guide Mr. Jitendra Dangra . I would also like to acknowledge Mr. M. K Rawat (H.O.D ), for their support and guidance during my research work.

\section{FUTURE WORK}

This proposed work describes wireless sensor networks and implementation of rapid sample algorithm and its extension used for reliable end to end communication. WSN is a sort range wireless communication system which is promises to provide reliable communication at optimum speed. WSN networks are involves a large amount of applications and implementation benefits.In near future we work under same concept of clustering of WSN communication and enhance more for cluster organization, cluster head dynamically selection, and rang improvements on WSN network.

\section{REFERENCES}

[1] Lenin Ravindranath, Calvin Newport, HariBalakrishnan Samuel Madden, Improving Wireless Network Performance using External Sensors ,In NSDI 2011.

[2] LvayloHaratehere V, KoenLangendoen , Reginald Lagendijk, Henksips, " Hybrid Rate control for IEEE 802.11 , Netherlands

[3] Steven Lanzisera ,Ankur M. Mehta and S.J Pister, Reducing Average Power in Wireless Sensor Network.

[4] .J. C. Bicket, "Bit-rate Selection in Wireless Networks," Master's thesis, Massachusetts Institute of Technology, 2005
[5] AdityaGudipati, SachinKatti, "Automatic Rate Adaptation" in Info System Lab, Stanford University [6]AbhishekMajumdar, NityanandaSarma , DEMAC Cluster Based Topology Control for AdHoc Networks , IJCSI 2010

[6] SaadBaiz ,Shaon Wu, "Rate Adaptation Algorithm for IEEE 802.11 Networks: A Survey and comparison , ISCC 2008. ieee symposium.

[7] http://www.cisco.com/en/US/solutions/collateral/ns341/n s525/ns537/ns705/ns827/white_paper_c11-520862.html

[8] AhrenStuder , Elaine Shi, Fan Bai, Adrian Perrig, "Tacking Together Efficient Authentication Revocation, and Privacy in VANET", CMU Cylab ,2008

[9] J. Kim, S. Kim, S. Choi, and D. Qiao, "CARA: CollisionAware Rate Adaptation for IEEE 802.11 WLANs," in Proceedings of IEEE INFOCOM, 2006.

[10] http://www.nsnam.org/

[11] MadhulikaMalviya, JitendraDangra ,M.K Rawat , "Proposed approach to optimize Rapid Sample Protocol ‘s Performance in Wireless Scenario”, IJCTM June 2013.

[12] Hartenstein, H. and Laberteaux, K. P. (eds) (2009) Introduction, in VANET: Vehicular Applications and Inter-Networking Technologies, John Wiley \& Sons, Ltd, Chichester, UK.[14] Shaoen Wu, Saad Biaz, Loss differentiated Rate Adaptation in Wireless Networks, Tech Report CSSE 07-02 2007. 